\title{
SELEÇÃO SIMULTÂNEA EM PROGÊNIES DE AÇAIZEIRO IRRIGADO PARA PRODUÇÃO E PESO DO FRUTO ${ }^{1}$
}

\author{
JOÃO TOMÉ DE FARIAS NETO², MARCOS DEON VILELA DE RESENDE, \\ MARIA DO SOCORRO PADILHA DE OLIVEIRA ${ }^{2}$
}

RESUMO - Para obtenção de materiais superiores, é necessário que o genótipo selecionado reúna, simultaneamente, uma série de atributos favoráveis que satisfaçam às exigências dos consumidores, produtores e processadores. Foram analisadas 50 progênies de açaizeiro conduzidas em dois delineamentos tipo látice, com duas repetições e cinco plantas por parcela, por um período de três safras, para as características de produção e peso de cem de frutos. O objetivo deste trabalho foi estimar parâmetros genéticos e fenotípicos e realizar a predição de valores genéticos dos indivíduos e progênies de açaizeiro irrigado no Estado do Pará, visando à seleção e ao melhoramento genético simultâneo para as características citadas, empregando a metodologia REML/BLUP. Foram empregados na seleção e na estimativa dos ganhos preditos, os índices com base na média de postos (ranks) e níveis independentes de eliminação, empregando uma intensidade de seleção de $20 \%$. Foi obtido ganho genético de 20,76 \% em relação à média do experimento com a seleção dos 20 melhores indivíduos para produção de frutos. Acima de cinco medições ou safras na avaliação de progênies de açaizeiro para produção de frutos, pouco acrescentará em termos de eficiência seletiva, sendo então injustificado seu uso. O índice com base na média de postos (rank) revelou-se o mais eficiente em selecionar, simultaneamente, progênies superiores para produção e menor peso de frutos, sendo recomendado seu uso em programa de melhoramento genético do açaizeiro.

Termos para indexação: Euterpe oleracea, melhoramento, índices de seleção, parâmetros genéticos, REML/ BLUP.

\section{SIMULTANEOUS SELECTION IN PROGENIES OF Euterpe oleracea IRRIGATED FOR FRUIT PRODUCTION AND WEIGHT}

\begin{abstract}
To obtain superior materials it is necessary that the selected genotype fulfills many favorable attributes that satisfy the demands of the consumers, the producers and the processors. Thus, data on the agronomic characteristics for fruit production and weight, involving 50 progenies of Euterpe oleracea were evaluated in a two type lattice designs with two replications and five plants per plot for a period of three seasons. The objective was to estimate genetic and phenotypic parameters and predicting breeding values of individuals and progeny of irrigated Euterpe oleracea in the State of Para aiming the simultaneous selection and breeding using the REML/BLUP methodology. For the selection and estimation of the predicted gains, it was used the indexes based on average ranks and independent levels of elimination, considering a selection intensity of $20 \%$. Genetic gain of $20.76 \%$ over the average of the experiment was obtained with the selection of the 20 best individuals for fruit production. Over five seasons in the measurement or evaluation of progenies of açai fruit production a little would be added in terms of selection efficiency, being their use unjustified. The index based on average ranks proved the most efficient both in selecting superior progenies for yield and lower fruit weight, being recommended its use in breeding program for açai.
\end{abstract}

Index terms: Euterpe oleracea, breeding, selection indexes, quantitative genetics, REML/BLUP.

'(Trabalho 146-10). Recebido em: 08-06-2010. Aceito para publicação em: 21-12-2010.

2Embrapa Amazônia Oriental. Caixa Postal 48.66105-100. Belém -PA. E-mails: tome@cpatu.embrapa.br; spadilha@cpatu.embrapa.br ${ }^{3}$ Embrapa Florestas, Colombo - PR. E-mail: marcos.deon@gmail.com 


\section{INTRODUÇÃO}

Com a expansão do mercado nacional e internacional de açaí, além do manejo de áreas de várzeas, o plantio está expandindo-se em áreas de terra firme, em antigas áreas de pimentais (Piper nigrum) e de outras lavouras abandonadas. Estes plantios envolvem consórcios com outras espécies perenes, como cacaueiro (Theobroma cacao), cupuaçuzeiro (Theobroma grandiflorum), pimenta-do-reino, etc., como etapa final de cultivos, tais como maracujazeiro (Passiflora edulis), bananeira (Musa spp.) etc., e também aproveitando áreas de pastagens degradadas. Nesse sentido, a mesorregião do nordeste paraense tem despertado a atenção dos produtores no plantio de açaizeiros, muitos deles, procurando inovar técnicas de cultivo, visando a desenvolver sistemas de cultivos apropriados, aumentar a produtividade e a produção, tanto na safra como na entressafra (HOMMA et al., 2009). Estima-se que $70-80 \%$ da produção de frutos ocorra no período de julho a dezembro, período considerado como safra (DIMENSTEIN; FARIAS NETO, 2008).

Os programas de melhoramento vegetal têm como objetivo a seleção de cultivares que aliem alta produtividade a outros caracteres desejáveis pelos agricultores, processadores e consumidores. No melhoramento genético do açaizeiro, a estratégia utilizada é a instalação e seleção de progênies de população aberta, normalmente baseada em apenas um caráter: a produção de frutos. Entretanto, a avaliação de apenas um caráter pode não ser adequado para representar o mérito econômico de uma planta, pois pode resultar no desenvolvimento de tipos economicamente insatisfatórios, seja pela não consideração de outros caracteres de importância econômica, seja pelas correlações negativas com estes (RESENDE, 2002). Na cultura do açaí, além da seleção para alta produtividade de frutos, fato que beneficia todos os atores da cadeia produtiva da cultura, os processadores interesse por frutos pequenos, que em função do maior número de frutos por peso e da maior área de processamento rende maior volume de suco. Diferenças genéticas têm sido detectadas para peso de frutos em açaizeiro, fato que indica haver possibilidade de ganhos genéticos via seleção (FARIAS NETO et al., 2008).

A seleção por meio de índices de seleção vêm sendo utilizada com sucesso no melhoramento de várias espécies frutíferas, dentre as quais se podem citar: guaraná (ATROCH, 2009), acerola (PAIVA et al., 2002), caju (PAIVA et al., 2007). Apesar da enfatizada potencialidade dos índices de seleção no melhoramento vegetal, em açaizeiro, nenhuma referência bibliográfica foi encontrada na literatura sobre o tema.

Realizou-se este trabalho com o objetivo de estimar parâmetros genéticos e fenotípicos e realizar a predição de valores genéticos dos indivíduos e progênies do programa de melhoramento genético do açaizeiro irrigado em andamento na Embrapa Amazônia Oriental, Estado do Pará, e o emprego dos métodos seletivos com base na média de postos e níveis independentes de eliminação, visando à seleção e ao melhoramento genético simultâneo da produção e peso de frutos, empregando a metodologia REML/BLUP (máxima verossimilhança restrita/ melhor predição linear não viciada).

\section{MATERIAL E MÉTODOS}

O estudo foi realizado na base experimental da Embrapa Amazônia Oriental, localizada no município de Tomé-Açu, no nordeste paraense, aproximadamente entre as latitudes de $01^{\circ} 5738^{\prime \prime}$ e $03^{\circ} 16^{\prime} 37^{\prime \prime}$ $\mathrm{S}$ e as longitudes de $47^{\circ} 53^{\prime} 32^{\prime \prime} \mathrm{W}$ e $48^{\circ} 49^{\prime} 15 \mathrm{~W}$. Os parâmetros meteorológicos climáticos indicam clima quente e úmido, ajustando-se ao tipo climático Ami, da classificação de Köeppen. A precipitação pluviométrica é caracterizada por dois períodos distintos de chuvas, dezembro a maio, com índices superiores a $150 \mathrm{~mm} / \mathrm{mês}$, onde se concentram cerca de $80 \%$ do total anual de precipitação, e outro, de junho a novembro, com índice variando de $49 \mathrm{~mm}$ a $105 \mathrm{~mm} / \mathrm{mês}$.

Os experimentos foram estabelecidos em março de 2003, em 2 látice 5 x 5, com duas repetições e 5 plantas por parcela, no espaçamento de $5 \mathrm{~m} \times 5 \mathrm{~m}$, totalizando 50 progênies, além de uma bordadura externa ao experimento sob irrigação. Os tratamentos foram representados por progênies de polinização livre, originadas de plantas selecionadas nos municípios de Afuá e Chaves, região norte da Ilha de Marajó. Essa região apresenta como principal característica a produção de frutos em período considerado como entressafra, ou seja, no primeiro semestre.

Na seleção fenotípica das árvores em condições naturais, utilizaram-se como critérios de seleção o número de cachos por planta,o tamanho do cacho, a presença de perfilhos e o estado fitossanitário das plantas.

A avaliação das progênies para os caracteres peso total dos frutos e peso médio de cem frutos teve início em agosto de 2006, quando as plantas estavam com 3,5 anos, indo até dezembro de 2007, representando a primeira safra. A segunda safra correspondeu ao período de janeiro a dezembro de 
2008, e a terceira, de janeiro a dezembro de 2009.

Para a análise dos dados, foi empregada a metodologia de modelos lineares mistos tipo REML/ BLUP (máxima verossimilhança restrita/melhor predição linear não viciada), para o delineamento de blocos incompletos desbalanceados. Isto permitiu o ajuste para os gradientes ambientais de blocos e também a recuperação de informação genética interblocos incompletos, propiciando eficiente estimação de parâmetros e seleção em nível de indivíduos. Os efeitos de blocos foram considerados como aleatórios, visando a propiciar a recuperação de informação genética interblocos. A metodologia REML/BLUP produz, simultaneamente, três tipos de informação: estimativas dos efeitos genéticos (e consequentemente do ganho ou progressão genética), estimativas dos efeitos ambientais e estimativas dos componentes de variância genética e ambiental (parâmetros genéticos).

Os parâmetros genéticos foram estimados via REML, e os genéticos aditivos individuais foram estimados pelo procedimento BLUP, por meio do software genético-estatístico Selegen-Reml/Blup (RESENDE, 2002).

Utilizou-se do seguinte modelo estatístico para a avaliação genética (RESENDE, 2002):

$\mathrm{y}=\mathrm{Xm}+\mathrm{Za}+\mathrm{Wp}+\mathrm{Tb}+\mathrm{Qc}+\mathrm{e}$, em que:

$\mathrm{y}, \mathrm{m}, \mathrm{a}, \mathrm{p}, \mathrm{b}, \mathrm{c}, \mathrm{e}=$ vetores de dados, de efeitos de medições (fixos), de efeitos genéticos aditivos individuais (aleatórios), de efeitos permanentes de indivíduo (aleatório), de efeitos de blocos (aleatórios), de efeito de parcelas (aleatório) e de erros aleatórios, respectivamente.

$\mathrm{X}, \mathrm{Z}, \mathrm{W}, \mathrm{T}$ e $\mathrm{Q}=$ matrizes de incidência para $\mathrm{m}, \mathrm{a}, \mathrm{p}, \mathrm{b}$ e c, respectivamente.

Associados a esse modelo, têm-se as seguintes distribuições e estruturas de médias e variâncias:

$$
\begin{aligned}
& y \mid m, V \sim N(X m, V) \\
& a \mid A, \sigma_{a}^{2} \sim N\left(0, A \sigma_{a}^{2}\right) \\
& p \mid \sigma_{p}^{2} \sim N\left(0, I \sigma_{p}^{2}\right) \\
& b \mid \sigma_{b}^{2} \sim N\left(0, I \sigma_{b}^{2}\right) \\
& c \mid \sigma_{c}^{2} \sim N\left(0, I \sigma_{c}^{2}\right) \\
& e \mid \sigma_{e}^{2} \sim N\left(0, I \sigma_{e}^{2}\right)
\end{aligned}
$$

As covariâncias entre todos os efeitos aleatórios do modelo são consideradas nulas. Assim:

$$
E\left[\begin{array}{c}
y \\
a \\
p \\
b \\
c \\
e
\end{array}\right]=\left[\begin{array}{c}
X m \\
0 \\
0 \\
0 \\
0 \\
0
\end{array}\right] \text { eVar }\left[\begin{array}{c}
y \\
a \\
p \\
b \\
c \\
e
\end{array}\right]=\left[\begin{array}{cccccc}
V & Z G & W P & T B & Q C & R \\
G Z^{\prime} & G & 0 & 0 & 0 & 0 \\
P W^{\prime} & 0 & P & 0 & 0 & 0 \\
B T^{\prime} & 0 & 0 & B & 0 & 0 \\
C Q^{\prime} & 0 & 0 & 0 & C & 0 \\
R & 0 & 0 & 0 & 0 & R
\end{array}\right] \text {, em que: }
$$

$$
\begin{aligned}
& G=A \sigma_{A}^{2} \\
& P=I \sigma_{p}^{2} \\
& B=I \sigma_{b}^{2} \\
& C=I \sigma_{c}^{2} \\
& R=I \sigma_{e}^{2} \\
& V=Z A \sigma_{a}^{2} Z^{\prime}+W I \sigma_{p}^{2} W^{\prime}+T^{\prime} I \sigma_{b}^{2} T+Q I \sigma_{c}^{2} Q^{\prime}+I \sigma_{e}^{2}=Z G Z^{\prime}+W P W^{\prime}+T B T^{\prime}+Q C Q^{\prime}+R .
\end{aligned}
$$


As equações de modelo misto para a predição BLUP dos valores genéticos individuais equivalem a:

$$
\left[\begin{array}{c}
\hat{b} \\
\hat{a} \\
\hat{p} \\
\hat{b} \\
\hat{c}
\end{array}\right]=\left[\begin{array}{ccccc}
X^{\prime} X & X^{\prime} Z & X^{\prime} W & X^{\prime} T & X^{\prime} Q \\
Z^{\prime} X & Z^{\prime} Z+A^{-1} \lambda_{1} & Z^{\prime} W & Z^{\prime} T & Z^{\prime} Q \\
W^{\prime} X & W^{\prime} Z & W^{\prime} W+I \lambda_{2} & W^{\prime} T & W^{\prime} Q \\
T^{\prime} X & T^{\prime} Z & T^{\prime} W & ' T T I \lambda_{3} & T^{\prime} Q \\
Q^{\prime} X & Q^{\prime} Z & Q^{\prime} W & Q^{\prime} T & { }^{\prime} Q+Q \lambda_{4}
\end{array}\right]^{-1}\left[\begin{array}{c}
X^{\prime} y \\
Z^{\prime} y \\
W^{\prime} y \\
T^{\prime} y \\
Q^{\prime} y
\end{array}\right] \text {, em que: }
$$

$\lambda_{1}=\frac{\sigma_{e}^{2}}{\sigma_{a}^{2}}=\frac{1-h^{2}-p^{2}-c^{2}-b^{2}}{h^{2}} ; \quad \lambda_{2}=\frac{\sigma_{e}^{2}}{\sigma_{p}^{2}}=\frac{1-h^{2}-p^{2}-c^{2}-b^{2}}{p^{2}} \quad \lambda_{3}=\frac{\sigma_{e}^{2}}{\sigma_{b}^{2}}=\frac{1-h^{2}-p^{2}-c^{2}-b^{2}}{b^{2}} ; \quad \lambda_{4}=\frac{\sigma_{e}^{2}}{\sigma_{c}^{2}}=\frac{1-h^{2}-p^{2}-c^{2}-b^{2}}{c^{2}}$ $h^{2}=\frac{\sigma_{a}^{2}}{\sigma_{a}^{2}+\sigma_{p}^{2}+\sigma_{c}^{2}+\sigma_{e}^{2}+\sigma_{b}^{2}}:$ herdabilidade individual no sentido restrito.

$p^{2}=\sigma_{p}^{2} /\left(\sigma_{a}^{2}+\sigma_{p}^{2}+\sigma_{c}^{2}+\sigma_{e}^{2}+\sigma_{b}^{2}\right):$ coeficiente de determinação dos efeitos de ambiente permanente.

$c^{2}=\sigma_{c}^{2} /\left(\sigma_{a}^{2}+\sigma_{p}^{2}+\sigma_{c}^{2}+\sigma_{e}^{2}+\sigma_{b}^{2}\right)$ : correlação devida ao ambiente comum da parcela.

$b^{2}=\sigma_{b}^{2} /\left(\sigma_{a}^{2}+\sigma_{p}^{2}+\sigma_{c}^{2}+\sigma_{e}^{2}+\sigma_{b}^{2}\right)$ correlação devida ao ambiente comum do bloco dentro de repetição.

$\sigma_{\mathrm{a}}^{2}$ : variância genética aditiva.

$\sigma_{p}^{2}$ : variância de ambiente permanente entre indivíduos.

$\sigma_{\mathrm{c}}^{2}$ : variância entre parcelas.

$\sigma_{b}^{2}$ : variância entre blocos.

$\sigma_{\mathrm{e}}^{2}$ : variância residual.

A: matriz de correlação genética aditiva entre os indivíduos em avaliação.

$\hat{\sigma}_{e}^{2}=\left[y^{\prime} y-\hat{m}^{\prime} X^{\prime} y-\hat{a}^{\prime} Z^{\prime} y-\hat{p}^{\prime} W^{\prime} y-\hat{b}^{\prime} T^{\prime} y-\hat{c}^{\prime} Q^{\prime} y\right] /[N-r(X)]$

$\hat{\sigma}_{a}^{2}=\left[\hat{a}^{\prime} A^{-1} \hat{a}+\hat{\sigma}_{e}^{2} \operatorname{tr}\left(A^{-1} C^{22}\right)\right] / q$

$\hat{\sigma}_{p}^{2}=\left[\hat{p}^{\prime} \hat{p}+\hat{\sigma}_{e}^{2} \operatorname{tr} C^{33}\right] / q$

$\hat{\sigma}_{c}^{2}=\left[\hat{c}^{\prime} c+\hat{\sigma}_{e}^{2} \operatorname{tr} C^{44}\right] / s$

$\hat{\sigma}_{b}^{2}=\left[\hat{b}^{\prime} \hat{b}+\hat{\sigma}_{e}^{2} \operatorname{tr} C^{55}\right] / \eta$

$C^{22}, C^{33}, C^{44}$ a $C^{55} \quad$, em que:

$C^{22}, C^{33}, C^{44}$ a $C^{55}$ advêm da inversa da matriz dos coeficientes das equações de modelo misto. tr: operador traço matricial.

$\mathrm{r}(\mathrm{X})$ : posto da matriz $\mathrm{X}$.

$\mathrm{N}, \mathrm{q}, \mathrm{s}, \eta$ : número total de dados, de indivíduos, de parcelas e de blocos, respectivamente. 
A seleção com base no índice de classificação (MULAMBA; MOCK, 1978), foi realizada com base nas três safras, considerando a média dos postos do desempenho de cada progênie para os caracteres produção de frutos e peso de cem sementes. Para a produção de frutos, a ordenação foi decrescente, ou seja, a seleção foi para progênies com maior produtividade, enquanto para o peso de cem frutos foi crescente, isto é, a seleção foi para progênies de menor peso. Na seleção baseada nos níveis independentes de eliminação adotou-se como nível mínimo de eliminação, a média populacional da produção de frutos, selecionando-se as progênies cujo desempenho a superava e com peso de cem frutos inferior a 130 gramas.

\section{RESULTADOS E DISCUSSÃO}

As estimativas dos parâmetros genéticos e fenotípicos para a análise conjunta, para o caráter produção de frutos, são apresentadas na Tabela 1. Constatou-se que o coeficiente de herdabilidade estimado no sentido restrito para o caráter apresentou magnitude baixa $(0,1233)$, de acordo com a classificação descrita por Resende (2002) onde a herdabilidade pode ser considerada como de baixa magnitude quando $\mathrm{h}^{2}<0,15$, média magnitude entre $0,15<\mathrm{h}^{2}>0,50$ e alta magnitude $\mathrm{com}^{2}{ }_{\mathrm{a}}>0,50$. A magnitude do valor obtido para herdabilidade mostra que um controle genético moderado pode ser obtido, o que garante que a seleção, em níveis de indivíduos e progênies, propiciaria ganhos significativos para o caráter. Em açaizeiro, Farias Neto et al. (2007) relatam herdabilidades variando de $12 \%$ a $44,63 \%$. $\mathrm{O}$ coeficiente de determinação dos efeitos de parcelas foi baixo $(0,0988)$, indicando a presença de uma pequena variação ambiental dentro das parcelas, ou seja, o efeito de parcelas foi pequeno. $\mathrm{O}$ coeficiente de determinação dos efeitos permanentes foi de média magnitude, revelando que a variação ambiental de um ano para outro é importante.

Na Tabela 2, são apresentados os coeficientes de repetibilidade e a eficiência seletiva para produtividade de frutos em relação a apenas uma medição ou safra. Verifica-se um aumento substancial na eficiência seletiva e acurácia $(1,1327 ; 0,3978$ na segunda medição), respectivamente, a partir da segunda até a quinta medição $(1,2431 ; 0,4366)$, a partir da qual ocorreu certa estabilização dos seus valores. $\mathrm{O}$ coeficiente de determinação, que demonstra a confiabilidade do valor fenotípico em predizer o valor real dos genótipos, apresentou valor expressivo com cinco medições $(0,8636)$. Esse valor de confiabilidade é referenciado como elevado, uma vez que, segundo
Resende (2002), quando se seleciona um grupo de indivíduos, valores acima de $80 \%$ já podem ser considerados adequados. Em coqueiro (FARIAS NETO et al., 2009), número semelhante de medição foi obtido. $\mathrm{O}$ valor de repetibilidade obtido apresenta magnitude média, conforme classificação de Resende (2002).

Na Tabela 3, são apresentados os resultados referentes aos valores genéticos e ganhos genéticos associados aos vinte melhores indivíduos. Ganho genético na ordem de $20,76 \%$ acima da média da população foram estimados, confirmando o potencial da população para fins de melhoramento para o caráter considerado.

Conforme Resende (2002), quando a seleção envolve mais de um caráter, três sistemas de seleção podem ser adotados: seleção em "tandem", níveis independentes de eliminação e índice de seleção. No primeiro, seleciona-se para determinado caráter até atingir o nível desejado e, em seguida, para outros caracteres de interesse. No segundo, níveis mínimos são estabelecidos para cada característica, e todos os indivíduos abaixo deste nível são eliminados. No sistema índice de seleção, consideram-se simultaneamente todos os caracteres de interesse.

$\mathrm{Na}$ Tabela 4, são apresentados os ganhos genéticos com a seleção das dez melhores progênies associadas às características produção e peso de cem frutos, utilizando as metodologias do índice com base na média de postos (MULAMBA; MOCK, 1978) e níveis independentes de eliminação. Com base na avaliação de três safras, utilizando uma intensidade de seleção de $20 \%$, o método da média dos postos apresentou ganhos genéticos consideráveis de $95,40 \%$. Por outro lado, o método de níveis independentes de eliminação proporcionou ganhos menores de $68,87 \%$. Verifica-se que, dentre as dez progênies selecionadas pelo método de Mulamba e Mock (1978), oito coincidem com as progênies selecionadas pelo método de níveis independentes. 
TABELA 1 - Estimativas de parâmetros genéticos e fenotípicos associados ao caráter produção de frutos em açaizeiro (avaliado em gramas) obtidos na análise conjunta das três safras. Belém, Embrapa Amazônia Oriental, 2010.

\begin{tabular}{lc}
\hline Parâmetros & Produção de frutos \\
\hline Variância genética aditiva & 14420485,674 \\
Variância ambiental entre parcelas & 11554690,447 \\
Variância dos efeitos permanentes & 39342052,483 \\
Variância residual & 51572825,858 \\
Variância fenotípica individual & 116890054,462 \\
Herdabilidade individual no sentido restrito & 0,1233 \\
Repetibilidade & 0,5587 \\
Coeficiente de determinação dos efeitos de parcelas & 0,0988 \\
Coeficiente de determinação dos efeitos permanentes & 0,336 \\
Média geral & 19038,57 \\
\hline
\end{tabular}

TABELA 2 - Estimativas da herdabilidade individual no sentido restrito, repetibilidade individual, determinação fenotípica, acurácia seletiva e eficiência do uso de determinado número de safras em relação ao uso de apenas uma, para o caráter produção de frutos em açaizeiro. Belém, Embrapa Amazônia Oriental 2010.

\begin{tabular}{cccccc}
\hline Medições & $\begin{array}{c}\text { Herdabilidade da } \\
\text { Média de Safras }\end{array}$ & $\begin{array}{c}\text { Repetibilidade } \\
\text { Individual }\end{array}$ & $\begin{array}{c}\text { Determinação } \\
\text { Fenotípica } \\
\text { Permanente }\end{array}$ & $\begin{array}{c}\text { Acurácia da } \\
\text { Seleção } \\
\text { Genética }\end{array}$ & Eficiência \\
\hline 1 & 0,1233 & 0,5587 & 0,5587 & 0,3512 & 1,0000 \\
2 & 0,1582 & 0,5587 & 0,7169 & 0,3978 & 1,1327 \\
3 & 0,1747 & 0,5587 & 0,7916 & 0,4181 & 1,1902 \\
4 & 0,1843 & 0,5587 & 0,8351 & 0,4293 & 1,2225 \\
5 & 0,1906 & 0,5587 & 0,8636 & 0,4366 & 1,2431 \\
6 & 0,1951 & 0,5587 & 0,8837 & 0,4417 & 1,2575 \\
7 & 0,1983 & 0,5587 & 0,8986 & 0,4454 & 1,2681 \\
\hline
\end{tabular}

TABELA3 - Valores genéticos aditivos e ganho genético associados à seleção dos vinte melhores indivíduos (baseada em 3 safras) para o caráter produção de frutos em açaizeiro. Belém, Embrapa Amazônia Oriental, 2010

\begin{tabular}{ccccccc}
\hline Ordem & Família & Planta & Valor fenotípico médio & Efeito genético & $\begin{array}{c}\text { Ganho genético } \\
(\%)\end{array}$ & Nova Média \\
\hline 1 & 37 & 308 & 42960,66 & 4891,67 & 25,69 & 23930 \\
2 & 27 & 403 & 38827,00 & 4467,11 & 25,11 & 23709 \\
3 & 20 & 96 & 42178,00 & 4481,39 & 24,58 & 23520 \\
4 & 33 & 286 & 46626,66 & 4333,90 & 24,13 & 23633 \\
5 & 11 & 137 & 38208,66 & 4159,29 & 23,68 & 23546 \\
6 & 4 & 17 & 37646,66 & 4131,42 & 23,34 & 23483 \\
7 & 41 & 328 & 38923,00 & 4128,90 & 23,11 & 23438 \\
8 & 26 & 251 & 38612,33 & 3917,20 & 22,79 & 23378 \\
9 & 45 & 494 & 37541,00 & 3883,03 & 22,53 & 2327 \\
10 & 12 & 59 & 35798,66 & 3860,21 & 22,30 & 23284 \\
11 & 4 & 203 & 38106,66 & 3842,36 & 22,10 & 23248 \\
12 & 45 & 350 & 35930,00 & 3805,11 & 21,93 & 23214 \\
13 & 15 & 238 & 36383,00 & 3769,94 & 21,76 & 23183 \\
14 & 12 & 165 & 34537,00 & 3722,27 & 21,61 & 23153 \\
15 & 37 & 310 & 36365,00 & 3705,49 & 21,47 & 23125 \\
16 & 50 & 497 & 38894,66 & 3648,12 & 21,32 & 23098 \\
17 & 27 & 256 & 32722,33 & 3616,95 & 21,18 & 23072 \\
18 & 12 & 161 & 33579,33 & 3550,04 & 21,04 & 23045 \\
19 & 41 & 329 & 35604,66 & 3532,12 & 20,91 & 23020 \\
20 & 12 & 162 & 32859,66 & 3420,61 & 20,76 & 22992 \\
\hline
\end{tabular}


TABELA 4 - Estimativas de ganhos genéticos obtidos pelos métodos com base na média de postos e níveis independentes de eliminação em progênies de açaizeiro. Belém, Embrapa Amazônia Oriental, 2010 .

\begin{tabular}{|c|c|c|c|c|c|c|c|c|c|}
\hline \multicolumn{5}{|c|}{ Î́ndice com base em soma de postos } & \multicolumn{5}{|c|}{ Níveis independentes de eliminação } \\
\hline Ordem & Progênies & $\begin{array}{l}\text { Rank } \\
\text { médio }\end{array}$ & Ganho & $\begin{array}{c}\text { Ganho } \\
(\%)\end{array}$ & Ordem & Progênies & $\begin{array}{l}\text { Rank } \\
\text { médio }\end{array}$ & Ganho & $\begin{array}{c}\text { Ganho } \\
(\%)\end{array}$ \\
\hline 1 & 20 & 7,5 & 7,50 & 240,00 & 1 & 20 & 7,50 & 7,50 & 240,00 \\
\hline 2 & 47 & 11,0 & 9,25 & 175,67 & 2 & 47 & 11,0 & 9,25 & 175,67 \\
\hline 3 & 10 & 11,5 & 10,00 & 155,00 & 3 & 10 & 11,5 & 10,00 & 155,00 \\
\hline 4 & 4 & 12,0 & 10,50 & 142,85 & 4 & 4 & 12,0 & 10,50 & 142,80 \\
\hline 5 & 29 & 12,5 & 10,90 & 133,94 & 5 & 29 & 12,5 & 10,90 & 133,94 \\
\hline 6 & 14 & 13,5 & 11,33 & 125,00 & 6 & 14 & 13,5 & 11,33 & 125,00 \\
\hline 7 & 7 & 14,0 & 11,71 & 117,68 & 7 & 7 & 14,0 & 11,81 & 117,68 \\
\hline 8 & 37 & 14,0 & 12,00 & 112,50 & 8 & 37 & 14,0 & 12,00 & 112,50 \\
\hline 9 & 41 & 17,0 & 12,55 & 103,09 & 9 & 3 & 19,0 & 14,26 & 78,71 \\
\hline 10 & 40 & 17,5 & 13,05 & 95,40 & 10 & 31 & 20,5 & 15,10 & 68,87 \\
\hline
\end{tabular}

\section{CONCLUSÕES}

1-Ganhos genéticos de $22,76 \%$ para produção de frutos foram obtidos pela seleção dos 20 melhores indivíduos.

2-Acima de cinco medições ou safras na avaliação de progênies de açaizeiro pouco acrescentará em termos de eficiência seletiva, sendo então injustificado seu uso.

3-O índice com base na média de postos (rank) revelou-se o mais eficiente em selecionar, simultaneamente, progênies superiores para produção e peso de cem frutos, sendo recomendado seu uso em programas de melhoramento genético do açaizeiro.

\section{REFERÊNCIAS}

ATROCH, A.L. Avaliação e seleção de progênies de meios-irmãos de guaranazeiro (Paullinia cupana var. sorbilis (Mart.) Ducke), utilizando caracteres morfoagronômicos. 2009. 72 f. Tese (doutorado) - Universidade Federal do Amazonas, Instituto Nacional de Pesquisa da Amazônia, Manaus, 2009.

DIMENSTEIN, L.; FARIAS NETO, J. T. de. Dados preliminares para a produção de frutos em açaizeiros sob irrigação em terra firme no Estado do Pará. In: DIMENSTEIN, L.; FARIAS NETO, J.T. Irrigação e fertirrigação em fruteiras. Fortaleza: Instituto Frutal, 2008. p.139-144.
FARIAS NETO, J.T. de; LINS, P.M.P.; RESENDE, M.D.V.; MULLER A.A. Seleção genética em progênies híbridas de coqueiro. Revista Brasileira de Fruticultura, Jaboticabal, v. 31, p.190-196, 2009.

FARIAS NETO, J.T. de; RESENDE, M.D.V.D de; OLIVEIRA, M.S.P. de; NOGUEIRA, O.L.; FALCÃO, P.N.B.; SANTOS, N.S.A. Estimativas de parâmetros genéticos e ganhos de seleção em progênies de polinização aberta de açaizeiro. Revista Brasileira de Fruticultura, Jaboticabal, v. 30, p.1051-1056, 2008.

FARIAS NETO, J.T. de; RESENDE, M.D.V.; OLIVEIRA, M.S.P.; SANTOS, N.S.A.; CANUTO, E.L.; NOGUEIRA, O.L.; MÜLLER A. A. Avaliação genética de progênies de polinização aberta de açaí (Euterpe oleracea) e estimativas de parâmetros genéticos. Cerne, Lavras, v. 13, n.4, p.376-383, 2007.

HOMMA, A.K.O.; CARVALHO, J.E.U.; FARIAS NETO, J.T. de; MENEZES; A.J.E.A.; MATOS, G. B. Custo operacional de açaizeiro irrigado com microaspersão no município de Tomé-Açu. Belém: Embrapa Amazônia Oriental, 2009. 5p. (Comunicado Técnico, 88).

MULAMBA, N.N.; MOCK, J.J. Improvement of yield potential of the method Eto Blanco maize (Zea mays L.) population by breeding for plant traits. Egyptian Journal of genetics and Cytology, Alexandria, v.7, p. 40-51, 1978. 
PAIVA, J.R.; ALVES, R.E.; MELO, F.I.O.; CORDEIRO, E.R.; ALMEIDA, A.S. Genetic progress of selections between and within caribbean cherry open pollination progenies. Crop Breending and Applied Biotechnology, Londrina, v.2, p.299306, 2002.

PAIVA, J.R.; CAVALCANTA, J.J.V.; BARROS, L.M.; CORRÊA, M.C.M.; MAIA, M.C.C.; COSTA FILHO, A.B. Seleção de clones de cajueiro comum pelo método em tandem e índice de classificação. Ciência e Agrotecnologia, Lavras, v.3, p.765-772, 2007.
RESENDE, M.D.V. de. Genética biométrica e estatística no melhoramento de plantas perenes. Brasília: Embrapa Informação Tecnológica, 2002. 975p. 